\title{
MicroRNA-378b regulates $\alpha$-1-type 1 collagen expression via sirtuin 6 interference
}

\author{
DAHYE JOO ${ }^{1 *}$, SUNGKWAN AN $^{2 *}$, BYUNG GON CHOI $^{3}$, KARAM KIM $^{1}$, \\ YOUNG MIN CHOI ${ }^{2}, \mathrm{KYU}_{\mathrm{JOONG}} \mathrm{AHN}^{3}$, IN-SOOK $\mathrm{AN}^{1}$ and HWA JUN CHA ${ }^{4}$ \\ ${ }^{1}$ Korea Institute of Dermatological Sciences, Cheongju, Chungcheongbuk 361-951; ${ }^{2}$ Korea Institute for Skin and Clinical \\ Sciences, Konkuk University; ${ }^{3}$ Department of Dermatology, Konkuk University School of Medicine, Seoul 143-701; \\ ${ }^{4}$ Department of Beauty Care and Cosmetics, Osan University, Osan, Gyeonggi 18119, Republic of Korea
}

Received February 28, 2016; Accepted February 10, 2017

DOI: $10.3892 / \mathrm{mmr} .2017 .7657$

\begin{abstract}
Ultraviolet (UV) light mediates skin aging and induces destruction of the dermis by modulating the expression levels of extracellular matrix-associated genes, including collagen and matrix metalloproteinases. Sirtuin 6 (SIRT6), a member of the sirtuin family of proteins, regulates collagen metabolism and is an established anti-aging protein. However, the exact underlying mechanism by which SIRT6 expression is regulated in dermal fibroblasts during the aging process is unclear. The present study demonstrated that expression of microRNA-378b (miR-378b) is induced in UVB-exposed human dermal fibroblasts (HDFs), and this was inversely associated with the mRNA expression levels of $\alpha$-1-type 1 collagen (COL1A1). In addition, knockdown of miR-378b enhanced the mRNA expression levels of COL1A1 in HDFs. A target analysis for miR-378b was performed, and the results revealed that SIRT6, a regulator of COL1A1, contains a target sequence for miR-378b in its 3'untranslated region. Notably, the present study demonstrated that an miR-378b mimic and inhibitor may directly regulate SIRT6 expression in HDFs. In conclusion, the present study suggested that miR-378b represses the mRNA expression levels of COL1A1 via interference with SIRT6 in HDFs, and may contribute to the underlying molecular mechanism by which UVB inhibits collagen I in dermal fibroblasts.
\end{abstract}

\section{Introduction}

Skin aging is a biological process that, as for aging of other organs, is mediated by intrinsic and extrinsic factors. Features of skin aging include wrinkling, reduced elasticity, sagging,

Correspondence to: Dr Hwa Jun Cha, Department of Beauty Care and Cosmetics, Osan University, Heemang Building, 45 Cheonghak-ro, Osan, Gyeonggi 18119, Republic of Korea

E-mail: hjcha@osan.ac.kr

*Contributed equally

Key words: microRNA-378b, collagen, sirtuin 6 laxity, dullness, roughness and discoloration. Deep wrinkles, in particular, are a primary consequence of ultraviolet (UV)-mediated skin aging (1-3). Histological studies have revealed that a reduction in collagen is one of the primary features of UV-mediated skin aging (1-5). This occurs via degradation of collagen and the collagen matrix, in addition to factors that interfere with their synthesis $(3,6)$. Underlying this process are molecular mechanisms that modulate expression of collagen, matrix metalloproteinases and associated proteins (7).

The sirtuin (SIRT) family is composed of nicotinamide adenine dinucleotide-dependent protein deacetylases, which regulate aging in yeast, worms, flies and mammals $(8,9)$. In humans, seven SIRT genes (SIRT1-7) have been identified, and these serve roles in numerous age-associated diseases, including cancer, neurodegenerative diseases, diabetes and cardiovascular disease (9-12). In mice, SIRT1 deficiency induces destruction of the skin barrier and enhances UV-induced injury and sensitivity (10-12). Additionally, SIRT1 may promote collagen expression in human smooth muscle cells by the deacetylation of regulatory factor X5 and alleviation of $\alpha$-1-type 2 collagen (COL1A2) repression, and has been implicated in the inhibition of the transforming growth factor- $\beta$ /mothers against decapentaplegic signaling pathway in fibroblasts during fibrosis (13-15). In mice, a deficiency in SIRT6 led to a skin aging phenotype (16), and the expression levels of collagen 1 and 3 are reduced by knockdown of SIRT6 in dermal fibroblasts (16). Therefore, regulation of SIRT1 and SIRT6 is associated with skin aging.

MicroRNAs (miRNAs) are small non-coding RNAs that are primarily generated by RNA polymerases II and III, and are processed and matured by the proteins Drosha and Dicer $(17,18)$. These miRNAs consist of 20-24 nucleotides and associate with the miRNA-induced silencing complex. This complex targets the $3^{\prime}$ untranslated region (3'UTR) of specific target genes, which interferes with translation and therefore down-regulates protein expression $(19,20)$. miRNAs serve a role in numerous biological processes, including development, differentiation, the cell cycle, apoptosis, stemness and tumorigenesis (21-25).

In the present study, a novel miRNA that regulates SIRT6 was identified that may regulate collagen expression. 


\section{Materials and methods}

Cell culture. Human dermal fibroblasts (HDFs) were purchased from Lonza (Basel, Switzerland) and cultured in Dulbecco's modified Eagle's medium (HyClone; GE Healthcare Life Sciences, Logan, UT, USA) supplemented with $10 \%$ fetal bovine serum (Hyclone; GE Healthcare Life Sciences) and $1 \%$ penicillin and streptomycin. Cells were transfected with $100 \mathrm{nM}$ miRNA (miR)-378b mimic (5'-ACUGGACUUGGA GGCAGAA-3'; Bioneer Corporation, Dajeon, Korea), 100 nM anti-miR-378b (5'-UUCUGCCUCCAAUCCUGU-3'; Bioneer Corporation) or $100 \mathrm{nM}$ scrambled control (AccuTarget ${ }^{\mathrm{TM}}$ Negative Control siRNA; Bioneer Corporation) using Lipofectamine ${ }^{\circledR}$ RNAiMAX Transfection reagent (Invitrogen; Thermo Fisher Scientific, Inc., Waltham, MA, USA), according to the manufacturer's protocol. After transfection for $4 \mathrm{~h}$, cells were washed with PBS and incubated for $24 \mathrm{~h}$. Transfected HDFs were subsequently exposed to UVB using a Super light-VI UV illuminator (Boteck, Gunpo, Korea). After incubation for $24 \mathrm{~h}$ following UVB exposure, reverse transcription-quantitative polymerase chain reaction (RT-qPCR), western blotting and luciferase assay were performed.

RNA extraction and $R T-q P C R$. Total RNA was isolated from cells using TRIzol ${ }^{\circledR}$ reagent (Invitrogen; Thermo Fisher Scientific, Inc.). cDNA was synthesized using $2 \mu \mathrm{g}$ RNA with the miScript II RT kit (Qiagen GmbH, Hilden, Germany), according to the manufacturer's protocol. Following cDNA synthesis, the mRNA expression levels of COLIAl and the gene encoding $\beta$-actin were detected using the StepOnePlus Real-Time PCR system (Applied Biosystems; Thermo Fisher Scientific, Inc.), using EvaGreen ${ }^{\mathrm{TM}}$ premix (Solis BioDyne, Tartu, Estonia) and the following specific primers: Forward, 5'-AGGGCCAAGACATC-3' and reverse, 5'-AGA TCACGTCATCGCACAACA-3' for human COL1A1; and forward, 5'-GGATTCCTATGTGGGCGACGA-3' and reverse, 5'-CGCTCGGTGAGGATCTTCATG-3' for human $\beta$-actin. Expression levels of miR-378b were detected with miR-378b specific primers (Qiagen $\mathrm{GmbH}$ ) using the hsa-mir-378b miScript Primer assay (cat. no. MI0014154) and Hs_RNU6-2_11 miScript Primer assay (cat. no. MS00033740) from Qiagen $\mathrm{GmbH}$, and the miScript $\mathrm{SYBR}^{\circledR}$ Green PCR kit (Qiagen $\mathrm{GmbH}$ ) with the StepOnePlus Real-Time PCR system. Forward primers were included in the miScript Primer assay kits and reverse primers were included in the miScript SYBR ${ }^{\circledR}$ Green PCR kit. The expression levels of COL1A1 and miR-378b were normalized to $\beta$-actin and U6 respectively, using the $2^{-\Delta \Delta C q}$ method (26). All RT-qPCRs were performed as follows: Initialization step at $94^{\circ} \mathrm{C}$ for $5 \mathrm{~min}$, followed by 40 cycles (denaturing, $94^{\circ} \mathrm{C}$ for $30 \mathrm{sec}$; annealing, $60^{\circ} \mathrm{C}$ for $30 \mathrm{sec}$; polymerization, $72^{\circ} \mathrm{C}$ for $30 \mathrm{sec}$ ) and a final elongation step at $72^{\circ} \mathrm{C}$, for $5 \mathrm{~min}$. All experiments were repeated three times. Data was analyzed with Excel 2016 (Microsoft Corporation, Redmond, WA, USA) and presented as the mean value of viable cells \pm standard deviation.

Target prediction and identification of $m i R-378 \mathrm{~b}$. Predicted targets of miR-378b were identified using the bioinformatic analysis tool, microRNA.org (www.microrna.org). A luciferase reporter construct containing the predicted target sequence of
miR-378b in the SIRT6 3'UTR was generated by ligating a region $(+1,312$ to $+1,329)$ of the human SIRT6 gene into the $X b a \mathrm{I}$ restriction site, downstream of the luciferase gene in the pGL3 vector (Promega Corporation, Madison, WI, USA). HDFs were subsequently transfected with the $1 \mu \mathrm{g}$ reporter assay vector and the $0.2 \mu \mathrm{g} \mathrm{pSV}-\beta$-galactosidase control plasmid (Promega Corporation), with or without an miR-378b mimic or anti-miR-378b, using Lipofectamine RNAiMAX Transfection reagent (Invitrogen; Thermo Fisher Scientific) for $4 \mathrm{~h}$, and subsequently incubated at $37^{\circ} \mathrm{C}$ for $24 \mathrm{~h}$. After incubation for $24 \mathrm{~h}$, luciferase and $\beta$-galactosidase assays were performed using Luciferase Assay Reagent (Promega Corporation) and the $\beta$-galactosidase Detection kit II (Clontech Laboratories, Inc., Mountainview, CA, USA), according to the manufacturer's protocol. Luciferase results were normalized using $\beta$-galactosidase activity.

Western blot analysis. Cells were lysed in radioimmunoprecipitation assay buffer, containing $1 \%$ NP-40, $150 \mathrm{mM} \mathrm{NaCl}$, $10 \mathrm{mM}$ Tris- $\mathrm{HCl}$ (pH 8.0), 1 mM EDTA and complete protease inhibitor cocktail (Roche Diagnostics, Basel, Switzerland). Extracted proteins ( $20 \mu \mathrm{g}$ ) were loaded onto $12 \%$ gels and separated by electrophoresis. Proteins were subsequently transferred onto nitrocellulose membranes (EMD Millipore, Billerica, MA, USA) and blocked with blocking buffer [(5\% skim milk in TBS-Tween-20 buffer (50 mM Tris, $150 \mathrm{mM} \mathrm{NaCl,} \mathrm{0.1 \%} \mathrm{Tween}$ 20)] at $25^{\circ} \mathrm{C}$ for $1 \mathrm{~h}$. Protein expression levels of SIRT6 and $\beta$-actin were detected using rabbit anti-SIRT6 (1:2,000; D8D12; cat. no. 12486; Cell Signaling Technology, Inc., Danvers, MA, USA) anti- $\beta$-actin $(1: 10,000 ; \mathrm{N}-21$; cat. no. sc-130656; Santa Cruz Biotechnology, Inc., Dallas, TX, USA) primary antibodies, followed by anti-mouse IgG horseradish peroxidase (HRP)-conjugated (1:5,000; cat. no. 7076; Cell Signaling Technology, Inc.) and anti-rabbit IgG HRP-conjugated antibody (1;3,000; cat. no. 7074; Cell Signaling Technology, Inc.) secondary antibodies. Membranes were incubated with primary antibody at $25^{\circ} \mathrm{C}$ for $4 \mathrm{~h}$, followed by incubation with secondary antibody at $25^{\circ} \mathrm{C}$ for $1 \mathrm{~h}$. Proteins were visualized using SuperSignal ${ }^{\mathrm{TM}}$ West Pico Chemiluminescent Substrate (Thermo Fisher Scientific, Inc.). The intensity of each band was measured using ImageJ software Version 1.50 (National Institutes of Health, Bethesda, MD, USA).

Statistical analysis. Statistical significance was calculated using one-way analysis of variance with Tukey's post-hoc test. $\mathrm{P}<0.05$ was considered to indicate a statistically significant difference using Excel 2016 (Microsoft Corporation, Redmond, WA, USA). Data are presented as the mean \pm standard error.

\section{Results and discussion}

UVB exposure enhances miR-378b and reduces COL1A1 expression levels in HDFs. The expression levels of various miRNAs alter during skin aging and in UVB-exposed cells (27). Therefore, to determine whether miR-378b is modulated by UVB, miR-378b expression was measured by RT-qPCR in UVB-exposed HDFs. miR-378b expression levels were significantly enhanced in cells exposed to $5-25 \mathrm{~mJ} / \mathrm{cm}^{2} \mathrm{UVB}$ in a dose-dependent manner, compared to untreated cells $\left(0 \mathrm{~mJ} / \mathrm{cm}^{2}\right.$ UVB; $\mathrm{P}<0.05$; Fig. 1A). In addition, in HDFs exposed to the 

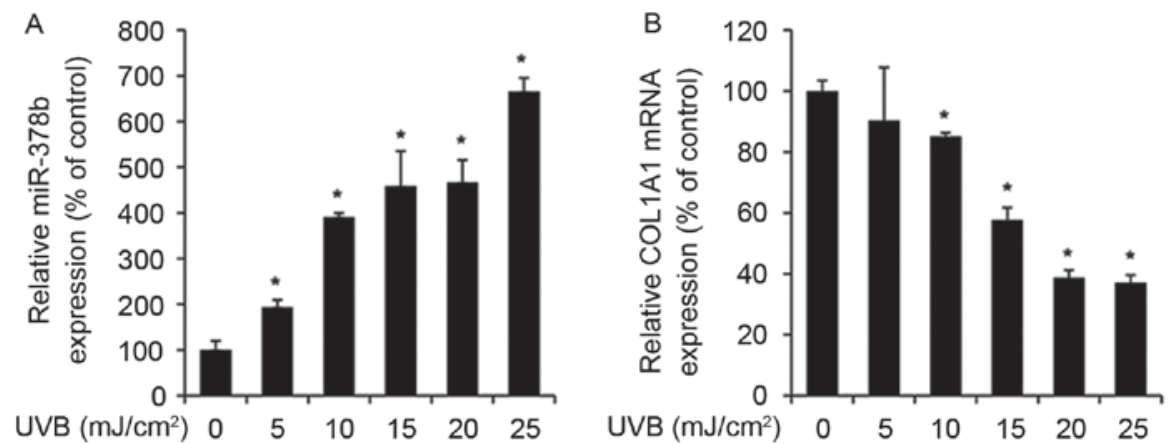

Figure 1. UVB treatment enhances miR-378b and reduces COL1A1 mRNA expression levels in HDFs. HDFs were exposed to various doses of UVB and reverse transcription-quantitative polymerase chain reaction was performed following a 24-h incubation. Expression levels of (A) miR-378b and (B) COL1A1. Data are expressed as the mean \pm standard error of three independent experiments, as percentage of the untreated control. ${ }^{*} \mathrm{P}<0.05 \mathrm{vs}$. untreated cells $\left(0 \mathrm{~mJ} / \mathrm{cm}^{2}\right)$. $\mathrm{UVB}$, ultraviolet B; miR-378b, microRNA-378b; COL1A1, $\alpha$-1-type 1 collagen; HDFs, human dermal fibroblasts.

A

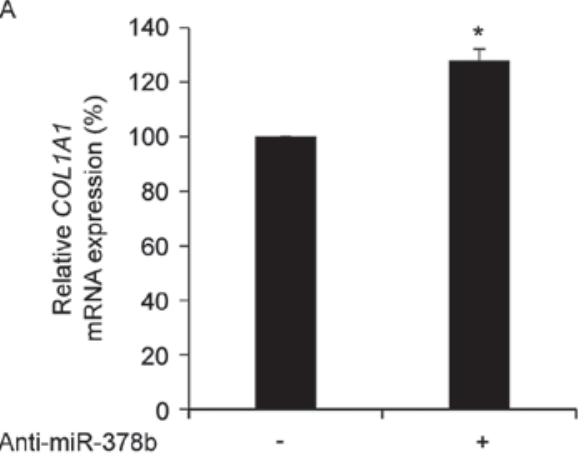

B

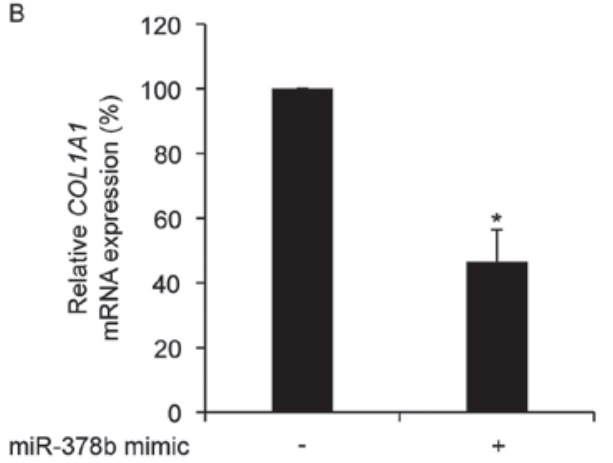

Figure 2. COL1A1 is negatively regulated by miR-378b in HDFs. HDFs were transfected with (A) anti-miR-378b or (B) an miR-378b mimic, and reverse transcription-quantitative polymerase chain reaction was performed to detect the mRNA expression levels of COL1A1. Data are expressed as the mean \pm standard error of three independent experiments, as a percentage of the scramble control-transfected cells. ${ }^{*} \mathrm{P}<0.05$ vs. scramble control. COL1A1, $\alpha-1$-type 1 collagen; miR-378b, microRNA-378b; HDFs, human dermal fibroblasts.

same doses of UVB, expression levels of COL1Al were reduced in a dose-dependent manner compared with untreated cells, and were inversely associated with miR-378b expression levels (Fig. 1B).

COL1A1 is negatively regulated by miR-378b in HDFs. To determine whether the expression of COL1A1 is directly affected by miR-378b, the mRNA expression levels of COL1A1 in HDFs treated with anti-miR-378b or an miR-387b mimic were measured. Transfection with anti-miR-378b significantly up-regulated COL1A1 mRNA expression levels $(\mathrm{P}<0.05$; Fig. 2A), whereas transfection with an miR-378b mimic resulted in a significant reduction in COL1A1 mRNA expression levels compared with cells transfected with a scramble control $(\mathrm{P}<0.05$; Fig. 2B). However, the 3'UTR of COL1A1 does not contain a predicted binding site for miR-378b. In addition, the miR-378b mimic did not directly modulate the luciferase activity of a luciferase-COL1A1 3'UTR fusion construct in HDFs (data not shown). Therefore, a target prediction for miR-378b was performed using microRNA.org, which revealed that SIRT6 contained a binding site for the miR-378b seed sequence in its 3'UTR. SIRT6 has been identified as an anti-aging protein due to its ability to regulate the mRNA expression levels of COLIAI and COL3A1 in HDFs (16). Therefore, it was hypothesized that miR-378b may directly regulate SIRT6 expression.
miR-378b negatively regulates SIRT6 by binding to its 3'UTR. The majority of miRNAs regulate target genes by binding to their 3'UTRs $(28,29)$. It was therefore determined whether the miR-378b mimic directly interacted with the SIRT6 3'UTR, by measuring the luciferase activity from a luciferase-SIRT6 3'UTR fusion construct. It was determined, using microRNA.org, that the seed sequence from miR-378b matched a region between $+1,312$ and $+1,324$ bp of the SIRT6 3'UTR (Fig. 3A). The SIRT6 3'UTR was subsequently cloned into a luciferase plasmid and transfected into HDFs. Co-transfection of an miR-378b mimic significantly reduced luciferase activity compared with pGL3-SIRT6-3'UTR only $(\mathrm{P}<0.05$; Fig. 3B), whereas co-transfection with an miR-378b mimic and anti-miR-378b significantly enhanced luciferase activity compared with cells transfected with SIRT6 3'UTR and an miR-378b mimic ( $\mathrm{P}<0.05$; Fig. 3B). Thus, these results suggested that miR-378b directly binds to, and interferes with, SIRT6 mRNA.

miR-378b represses endogenous SIRT6 expression in HDFs. It was subsequently determined whether the miR-378b mimic affected endogenous SIRT6 protein expression by western blot analysis. Overexpression of miR-378b reduced the expression levels of SIRT6 (Fig. 4A). Transfection with anti-miR-378b had the reverse effect and enhanced SIRT6 
A

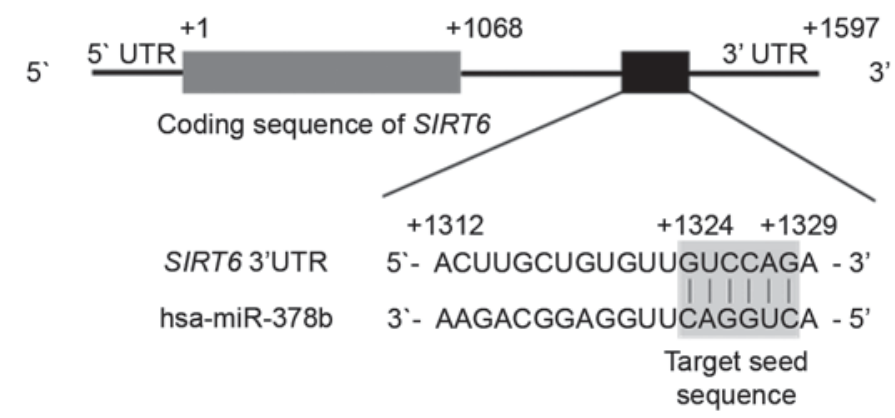

B

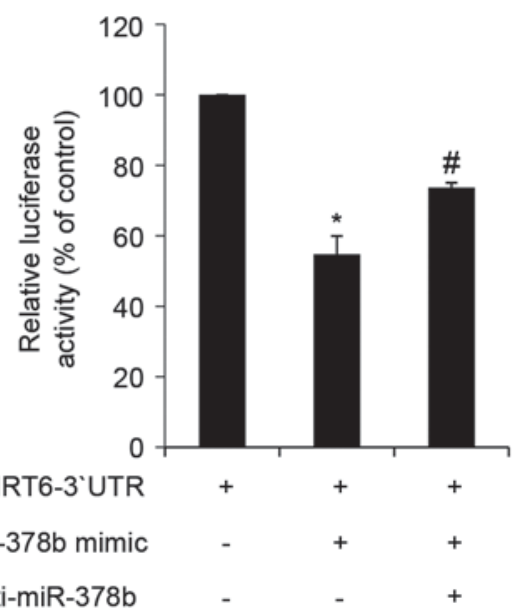

Figure 3. miR-378b directly targets SIRT6 mRNA and represses its translation. (A) In silico analysis of SIRT6 mRNA and the predicted target sequence of miR-378b. The ATG start codon is indicated by +1 , and the 3 ' end of the SIRT6 coding region is indicated by $+1,068$. The region of the 3 'UTR containing the miR-378b recognition sequences is located from $+1,312$ to $+1,329$ in the SIRT6 transcript. (B) HDFs were transfected with a reporter construct containing the miR-378b recognition sequences from SIRT6 fused to luciferase, and pSV- $\beta$-galactosidase, which was the control vector for normalization. Additional groups were co-transfected with an miR-378b mimic and anti-miR-378b. Following $24 \mathrm{~h}$ transfection, cells were harvested and luciferase assays were performed. Data are expressed as the mean \pm standard error of three independent experiments, as a percentage of the pGL3-SIRT6-3'UTR-transfected group. " $\mathrm{P}<0.05$ vs. pGL3-SIRT6-3'UTR only-transfected group. ${ }^{*} \mathrm{P}<0.05$ vs. pGL3-SIRT6-3'UTR and miR-378b mimic co-transfected group. miR-378b, microRNA-378b; SIRT6, sirtuin 6; UTR, untranslated region; HDFs, human dermal fibroblasts.
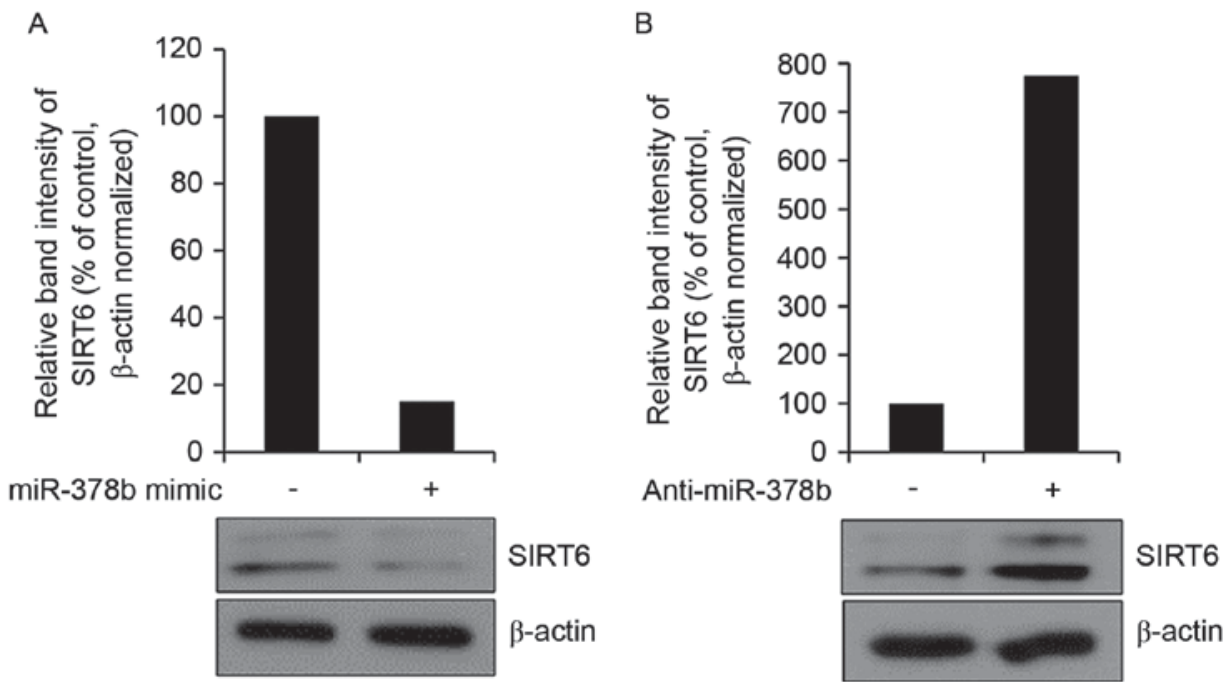

Figure 4. miR-378b downregulates protein expression levels of SIRT6 in HDFs. HDFs were transfected with (A) an miR-378b mimic or (B) anti-miR-378b, and western blot analysis was performed to detect SIRT6 protein expression levels. Image J analysis software was utilized for quantification of SIRT6 western blot band density, which was normalized to the $\beta$-actin loading control. Representative results from triplicate experiments are presented. miR-378b, microRNA-378b; SIRT6, Sirtuin 6; HDFs, human dermal fibroblasts.

protein expression levels (Fig. 4B). These results suggested that miR-378b may inhibit COL1A1 mRNA expression via effects on SIRT6. In addition, a previous study demonstrated that SIRT6 regulates the expression of genes associated with stress and aging (30). In HDFs from younger individuals, SIRT6 was highly activated compared with HDFs from older individuals (31). Therefore, miR-378b, which was upregulated by UVB in the present study (Fig. 1A), may regulate aging and collagen expression via interference of translation of SIRT6 mRNA. Investigation of the association between miR-378b and skin aging in human skin samples is required in future studies to indicate whether miR-378b is a marker of photo-aging.

\section{Acknowledgements}

The present study was supported by the Research Professor Program of Konkuk University (to Professor Hwa Jun Cha) and the Korean Health Technology R\&D Project, Ministry of Health \& Welfare, Republic of Korea (grant no. HN13C0075).

\section{References}

1. Lee YK, Cha HJ, Hong M, Yoon Y, Lee H and An S: Role of NF- $\kappa$ B-p53 crosstalk in ultraviolet A-induced cell death and G1 arrest in human dermal fibroblasts. Arch Dermatol Res 304: 73-79, 2012. 
2. Varani J, Dame MK, Rittie L, Fligiel SE, Kang S, Fisher GJ and Voorhees JJ: Decreased collagen production in chronologically aged skin: Roles of age-dependent alteration in fibroblast function and defective mechanical stimulation. Am J Pathol 168: 1861-1868, 2006.

3. Wulf HC, Sandby-Møller J, Kobayasi T and Gniadecki R: Skin aging and natural photoprotection. Micron 35: 185-191, 2004

4. Quan T and Fisher GJ: Role of age-associated alterations of the dermal extracellular matrix microenvironment in human skin aging: A mini-review. Gerontology 61: 427-434, 2015.

5. Darlenski R, Kazandjieva J and Tsankov N: Skin barrier function: Morphological basis and regulatory mechanisms. J Clin Med 4: 36-45, 2011.

6. Egbert M, Ruetze M, Sattler M, Wenck H, Gallinat S, Lucius R and Weise JM: The matricellular protein periostin contributes to proper collagen function and is downregulated during skin aging. J Dermatol Sci 73: 40-48, 2014.

7. Newton VL, Mcconnell JC, Hibbert SA, Graham HK and Watson RE: Skin aging: Molecular pathology, dermal remodelling and the imaging revolution. G Ital Dermatol Venereol 150 665-674, 2015.

8. Borradaile NM and Pickering JG: NAD (+), sirtuins, and cardiovascular disease. Curr Pharm Des 15: 110-117, 2009.

9. Law IK, Liu L, Xu A, Lam KS, Vanhoutte PM, Che CM Leung PT and Wang Y: Identification and characterization of proteins interacting with SIRT1 and SIRT3: Implications in the anti-aging and metabolic effects of sirtuins. Proteomics 9 : 2444-2456, 2009.

10. Han SH: Potential role of sirtuin as a therapeutic target for neurodegenerative diseases. J Clin Neurol 5: 120-125, 2009.

11. Roth M and Chen WY: Sorting out functions of sirtuins in cancer. Oncogene 33: 1609-1620, 2014

12. Balcerczyk A and Pirola L: Therapeutic potential of activators and inhibitors of sirtuins. Biofactors 36: 383-393, 2010.

13. Chou WW, Chen KC, Wang YS, Wang JY, Liang CL and Juo SH: The role of SIRT1/AKT/ERK pathway in ultraviolet B induced damage on human retinal pigment epithelial cells. Toxicol In Vitro 27: 1728-1736, 2013.

14. Fan W and Luo J: SIRT1 regulates UV-induced DNA repair through deacetylating XPA. Mol Cell 39: 247-258, 2010.

15. Xia J, Wu X, Yang Y, Zhao Y, Fang M, Xie W, Wang H and Xu Y: SIRT1 deacetylates RFX5 and antagonizes repression of collagen type I (COL1A2) transcription in smooth muscle cells. Biochem Biophys Res Commun 428: 264-270, 2012.

16. Baohua Y and Li L: Effects of SIRT6 silencing on collagen metabolism in human dermal fibroblasts. Cell Biol Int 36 : 105-108, 2012.
17. An IS, An S, Park S, Lee SN and Bae S: Involvement of microRNAs in epigallocatechin gallate-mediated UVB protection in human dermal fibroblasts. Oncol Rep 29: 253-259, 2013.

18. Lee MJ, Cha HJ, Lim KM, Lee OK, Bae S, Kim CH, Lee KH, Lee YN, Ahn KJ and An S: Analysis of the microRNA expression profile of normal human dermal papilla cells treated with 5 $\alpha$-dihydrotestosterone. Mol Med Rep 12: 1205-1212, 2015.

19. Ambros V: microRNAs: Tiny regulators with great potential. Cell 107: 823-826, 2001

20. Bartel DP: MicroRNAs: Genomics, biogenesis, mechanism, and function. Cell 116: 281-297, 2004.

21. Fahs F, Bi X, Yu FS, Zhou L and Mi QS: New insights into microRNAs in skin wound healing. IUBMB Life 67: 889-896, 2015.

22. Virant-Klun I, Ståhlberg A, Kubista M and Skutella T: MicroRNAs: From female fertility, germ cells, and stem cells to cancer in humans. Stem Cells Int 2016: 3984937, 2016.

23. Nothnick WB: Non-coding RNAs in uterine development, function and disease. Adv Exp Med Biol 886: 171-189, 2016.

24. Garg M: MicroRNAs, stem cells and cancer stem cells. World J Stem Cells 4: 62-70, 2012

25. Dumortier O and Van Obberghen E: MicroRNAs in pancreas development. Diabetes Obes Metab 14 (Suppl 3): S22-S28, 2012.

26. Livak KJ and Schmittgen TD: Analysis of relative gene expression data using real-time quantitative PCR and the 2(-Delta Delta C(T)) method. Methods 25: 402-408, 2001

27. Zhou BR, Xu Y and Luo D: Effect of UVB irradiation on microRNA expression in mouse epidermis. Oncol Lett 3: 560-564, 2012

28. Lewis BP, Burge CB and Bartel DP: Conserved seed pairing, often flanked by adenosines, indicates that thousands of human genes are microRNA targets. Cell 120: 15-20, 2005.

29. Chang YM, Juan HF, Lee TY, Chang YY, Yeh YM, Li WH and Shih AC: Prediction of human miRNAs using tissue-selective motifs in 3' UTRs. Proc Natl Acad Sci USA 105: 17061-17066, 2008.

30. Kawahara TL, Rapicavoli NA, Wu AR, Qu K, Quake SR and Chang HY: Dynamic chromatin localization of Sirt6 shapes stress- and aging-related transcriptional networks. PLoS Genet 7: e1002153, 2011.

31. Sharma A, Diecke S, Zhang WY, Lan F, He C, Mordwinkin NM, Chua KF and Wu JC: The role of SIRT6 protein in aging and reprogramming of human induced pluripotent stem cells. J Biol Chem 288: 18439-18447, 2013 